\title{
Implementation of Novel and Secured Parking Lot System using Image Hashing and Matlab
}

\author{
Mr. M. Sajid Mohammadi1,3, Dr. Syed Sohail Ahmed ${ }^{2}$, Dr.Syed khizer khasier ${ }^{3}$ \\ ${ }^{I}$ Department of Information Technology, College of Computer Qassim University,Buraidah, Saudi Arabia. \\ ${ }^{2}$ Department of Computer Engineering, College of Computer Qassim University, Buraidah, Saudi Arabia. \\ ${ }^{3}$ Departmentof Computer Science and Engineering, Noida International University J.B. Nagar, NCR Delhi, India.
}

\begin{abstract}
This paper proposes a management and implementation of Novel parking system in two folds, first based on cameras scanned image of parking lot and second Image Hashing technique for image or data security itself. As the parking problem become one of the key issue of concern to car drivers or motorists, it must be addressed in a good time saving way and secured way to give smooth or comfortable feel to the motorists as well as to the society as a whole. Our system will help motorists to know before entering in parking if there is a place available for their cars or not by show current parking status of parking places beforehand. Information of whether there is a parking free or not and how many parking are free at that point of time with its row - column address. The security to the system is provided by hashing algorithm. We used MATLAB software simulation to enable us to use different image prepossessing methods like detecting and tracking objects using separate background color from the other color objects by using Raspberry Pi microcontroller, Camera, module board, SD card that will increase rate of reliability and will decrease the cost. The Image hashing algorithms EfficientImageHashkeyGenerator will provide authenticity and security. Thus the proposed system will be secure system and support different operating Systems appropriate for various environments.
\end{abstract}

Keywords: Image hashing, Digital image processing; Efficient parking; MATLAB; Raspberry Pi.

\section{INTRODUCTION}

Digital image processing is pervasive, with applications ranging from television to tomography, from photography to printing, from robotics to remote sensing. Improves graphical or visual information for better human understanding and processing of image data for storage and transmission [1]. It also pervasive support in Digital Security Application etc. The purpose of this research is to produce an efficient and secured parking lot system using digital image hashing and image processing techniques. Efficient means that our system automatically counts the number of cars present in the lot and also tells us the number of vehicles that can more be parked. The basic technique which we used here is image subtraction.
Security in this application is provided explicitly by newly designed image hashing algorithm.

The normal vehicle wastes a total of 2,549 hours roaming the streets searching for an empty slot whether it is on the school side, the house street or a shop or an airport car park [1]. Knowing the availability parking is one of common problem to motorist. In this project we studied this problem and provided superlative solutions securely.

This problem exists because of long parking finding time but there are lots of solutions to this problem like: using sensors around gates to count number of cars parked inside the parking lot, one of gate increase counter number and other decrease it. Also, there are solutions embrace the idea of using sensor for each parking by using light sensor on ground of each parking and if car parked it's will cover parking and that will reduce lighting to some extent however, there was no idea or system that can ensure us as reliable system with low cost. Thus the objective of this research is to design a system that can give the drivers real time parking lot status or situation showing if there is a free parking or not? And how many parking is available. The main goal of our project is produce a proficient parking lot system using digital image processing techniques with MATLAB simulation software in implementation phases of the research work. Also use Image hashing techniques and Raspberry Pi camera to detect and track objects in parking lot to make this system reliable, inexpensive, and available all the time.

With consideration of these objective our important contribution in the system would be as follows.

\section{Our contribution:}

1. The 32-byte Hash key, Image hashing techniques has been introduced and designed to authenticate the image of parking lot thus the system can be trusted (reliability) and secured always.

2. It will be available all time (availability) hence our system is frugal, economical as uses cameras rather than sensors to get rid of high rate of large number of equipment.

3. By using several image processing techniques that include detecting and tracking objects that are more intelligent than using sensors is introduced; as the main problem of sensors is 
it can't know object in front of it whether object is car or bicycle or even human.

3. The system is available for all platform (Scope); it needs less equipment's (inexpensiveness is achieved)

The organization of the remaining paper is as follows, the next section II shows some literatures about hashing algorithm and some hardware parts, Section III introduces the proposed parking algorithm. Simulation is explained in the section IV. Section V conclude the work followed by limitations.

\section{LITERATURE REVIEW}

For this research we need to find the history in two fold one is for Image hashing algorithms and another is for automated electronic parking systems. In Part-A we will discuss literature about some image hashing techniques while in Part-B literature about some automated electronic parking systems is discussed.

\subsection{Part-A Literature about some image hashing techniques.}

Author Zhenjun Tang et al.in their work [2] has proposed a new approach to image hashing. Their basis was statistical weighted discrete wavelet transform (DWT) features. Using bilinear interpolation, they covert the image in to normalized image with color space conversion and extracted the edges with the use of famous Canny edge detection operator. And this edge frame image is divided into different blocks, for every block, 2 dimensional DWT is applied to get sub bands with their weighted sum. The image hash is generated by merging and quantizing these features. Euclidean distance is used to find the similarity among hashes. The authors also claim that they have better performance in image quality assessment than two popular measures, i.e. peak signal-to-noise ratio and structural similarity. The dataset used are CopyDays' dataset and the Uncompressed Color Image Database (UCID) are both used to evaluate classification between robustness and discrimination. while Live Image Quality database is used to check the algorithm in reduce reference image quality assessment.

The author in [3] has used the Multidimensional scaling (MDS) technique in case of image hashing algorithm design. They propose MDS hashing resistant to rotation of image by any angle by learning through MDS they could analysis compact, discriminative representation of image. The normalized image is used to extract the rotation invariant matrix features by using log polar transform and discrete Fourier transform. They experimented with more than 3800 different images and they claim that algorithm is outperforms in classification performance compared to some state-of-the-art techniques in robustness and discrimination.

This research [4] presented for $n$ integers better word based hash function they claim that this function is time independent. Here in this work, to process $n$ integers, it takes $\mathrm{O}(n)$ time.

The work in paper [5] the author has used one important feature of image Color Vector Angle (CVA), they generate image hashing algorithm with histogram of CVA feature in their efficient Image base hashing algorithm called HCVA hashing. In any normalized image the biggest angel in circle inscribed the histogram is first extracted from those to make a short hash after compression. Some experiments are conducted to find the performance. They found that for HCVA DCT (Discrete Cosine Transform) is the best suit for generation of hashes, as well as the HCVA hashing is robust and promising.

This work [6] proposes a robust image hashing algorithm. Here Normalized image is used to extract the image features using chaotic map called Skew tent map and Gabor filtering. With 2 DDWT it is compressed .The Image hash has been obtained in LL sub band DWT coefficient concatenations.

This paper [7] presented perceptual texture and structure features hashing method. The author followed the approach like, after preprocessing step the secondary image dual-cross pattern (DCP) is encoded and thus 2 coded map is generated. This represents image textual features information for all direction (horizontal-vertical and diagonal directions, respectively), using histogram composition the DCP-based textural features could be extracted. While frequency coefficient and its position information of chosen sampled block which has rich corner points is used to extract salient structural features. Thus after using dimension reduction of these two features types the final hash can be made.

\subsection{Part-B Literature about some automated electronic parking systems}

The novel parking system is developing in US, Europe and Japan by the cooperation of highly developed technologies and researches from various intellectual fields. Currently, there is a potential growth also speedy growth in parking lot system is observed. Human power is needed for each car parking space to select a parking slot manually and give route to drive properly into slot. So, there is a need to build up an automatic parking system which will lessen manual work as well as will be useful for careful parking of all types of vehicles.

While doing research the Authors A Kamble and A Dehankar have established that this autonomous car parking system has been wished-for by various researchers using different technology. In some research work, some researchers have proposed this system using Around View Monitor (AVM). In their manuscript they have discusses fusion of AVM and ultrasonic sensor, used to detect the free parking slot in the automatic car parking system [8].

While other researcher's K Sushma et al. have explained system like following by using different technology i.e. GSM Technology. The algorithm was working in the way that any user can send a message to a GSM modem at parking place and the modem will send availability of slot. The functionality of the technology is that user sends a message to the GSM modem which is placed at the parking end. If it is vacant the user has to message, the exact time and duration of parking slot needed. Then the GSM modem will send the parking lot number with password to access it. [9].

\subsection{Literature About Raspberry Pi microcontroller hardware kit features.}

\subsection{1: Raspberry Pi microcontroller:}

The Raspberry $\mathrm{Pi}$ is a small computer like microcontroller at low price, it can plug into any LCD screen or any monitor and works with any standard keyboard and mouse or connect remotely to use keyboard, screen and trackpad of laptop. It is a 
small but very useful piece of equipment could use to learn how to program codes in languages like Python and Scratch. It can do all jobs of that PC can do. It can communicate with outside world effectively hence used in verity of digital projects been used in a wide array of digital projects, from music machines and detectors to weather stations and tweeting birdhouses with infra-red camera. [10]

The Raspberry Pi hardware has evolved through several versions that feature variations in memory capacity, and peripheral device support. Fig.1 block diagram depicts models A, B, A+, and B+ in general. Model A and A+ lack the Ethernet and USB hub components. The Ethernet adapter is connected to an additional USB port. In model $\mathrm{A}$ and $\mathrm{A}+$ the USB port is connected directly to the SoC. On model $\mathrm{B}+$ the chip contains a five-point USB hub, of which four ports are available, while model B only provides two. [11]

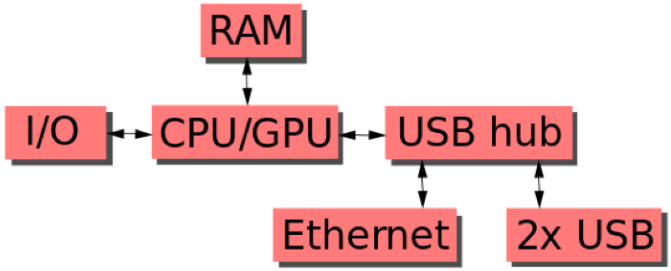

Figure 1: Raspberry Pi hardware

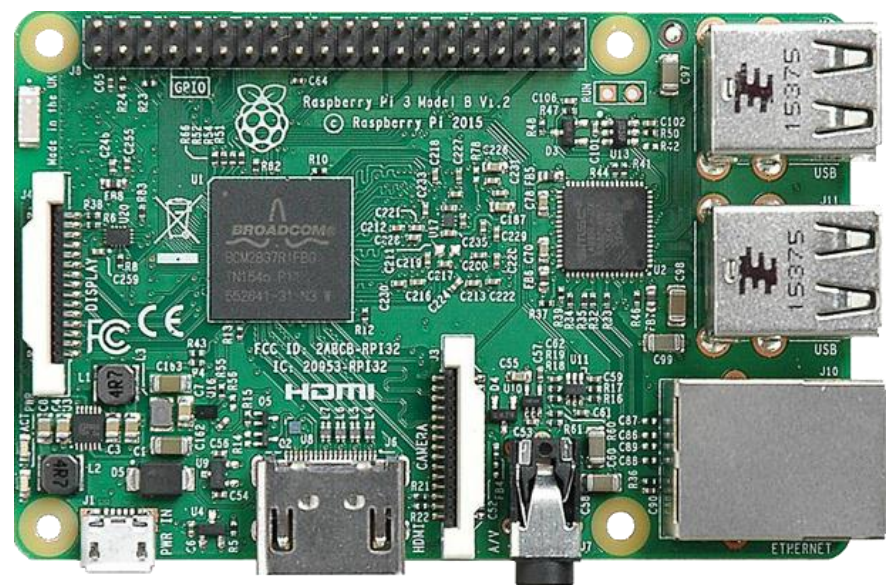

Figure 2: Raspberry PI 3 Model B

The Raspberry Pi 3 Model B in Figure 2 is the third-generation Raspberry Pi It replaced the Raspberry Pi 2 Model B in February 2016. [12] Features of Raspberry PI 3 Model B can be seen on website of Raspberry PI.

\subsubsection{Camera Module}

The Raspberry Pi Camera Module v2 shown in figure 3 replaced the original Camera Module v1 in April 2016. The v2 Camera Module has a Sony IMX219 8-megapixel sensor while 5-megapixel Omni Vision OV5647 sensor of the original camera $\mathrm{v} 1$.

The Raspberry Pi camera module v2 can be used to take highdefinition video, as well as stills photographs. It is capable of taking full HD photo and video and can be controlled programmatically.
The flex cable inserts into the connector situated between the Ethernet and HDMI ports, with the silver connectors facing the HDMI port. The flex cable connector should be opened by pulling the tabs on the top of the connector upwards then towards the Ethernet port.

The flex cable should be inserted firmly into the connector, with care taken not to bend the flex at too acute an angle. The top part of the connector should then be pushed towards the HDMI connector and down, while the flex cable is held in place [13].

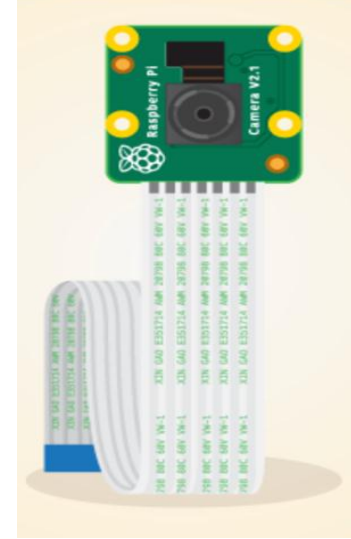

Figure 3: Camera Module 2 for Raspberry $\mathrm{Pi}$

\subsubsection{SD Flash Card}

The SD card is a key part of the Raspberry $\mathrm{Pi}$; it provides the initial storage for the Operating System and files. Storage can be extended through many types of USB connected peripherals. One advantage to using an SD card like this is that you can have several SD cards, each with a different operating system, or a different purpose. Simply power off, switch cards, and reconnect the power [15].

\subsubsection{Power}

The Raspberry Pi 3 is powered by a 5V micro USB supply. Exactly how much current $(\mathrm{mA})$ the Raspberry Pi requires is dependent on what you connect to it. We have found that purchasing a 2.4A (2400mA) power supply from a reputable retailer will provide you with ample power to run my Raspberry $\mathrm{Pi}$. The power requirements of the Raspberry Pi increase as you make use of the various interfaces on the Raspberry Pi $[15,16]$.

\section{NOVEL AND SECURED PARKINGLOT ALGORITHM}

In this Section we discuss why we need simulation? Then flow charts of both phases of novel hashing algorithm as well as parking lot algorithm system model, with elaboration of algorithm is given in detail.

Professionals in the field of designing system models have been creating models since many years. Sometimes the models designed are physical, such as scaled mock-ups of airplanes, vehicles and homes. Most of the times the models are less tangible as seen in different types of simulations as well as circuit diagrams. Basically a system model serves as a 
generalization and an estimated representation of the real item that is being built.

Is there any need for system modeling before they construct it? And why do they can't just fabricate the real thing in right direction? The solution has to do with the complication, the hazard and the fact that original practitioners are not always appropriate or even available for completing the task. It is neither theoretically intelligent nor cost-effectively method to build systems without first creating a design, a pictorial representation or another abstract representation. System modeling provides architects and others with the capacity to imagine entire systems, evaluate different options and correspond designs more clearly before taking on the risks technical, financial or otherwise of actual construction. In this research, we will talk about modeling and design system and describe how our system will work graphically using flowcharts and algorithms then we will give you our plan to implementation our project.

\subsection{Image Hashing}

The Figure 4 and figure 5 shows the flow charts of image hashing in two phases. In figure 4 it can be seen that the image is captured by any cameras. Our system supports many image format. The image could be of color or monochrome. It could be of JPEG or BMP or Tiff or RGB Image This image is firstly converted in to digital bit stream as a vector of zeros and ones [17]. Then the grouping is performed along these bit stream vector. Each group is of 7 bit in size. All seven bit of first group is performed Bitwise XOR operation with second group bits. Cumulatively till the last groups to from the first seed key Sk1, then second group is performed Bitwise XOR operation with third group bits Cumulatively till the last groups to form seed key Sk2. Similarly, first with third group and second with fourth group bits performed Bitwise XOR operation Cumulatively till the last but 1 groups to generate seed keys Sk3 and Sk4 respectively [17].

The generated key Sk1 to Sk4 are termed as seeds in key generation phase as shown in figure 5. In this phase we produce four groups of eight random numbers, as shown. The 4 sets of 8 char intermediate keys is produced by performing XOR operation again with their seeds respectively. After concatenation intermediate keys, image Hash Key is obtained which needs to be filter out by a filter to get proper output by removing special character from the Hash key. Hence we passed this 32-byte Image Hash key through it. There is a chance of changing $50 \%$ bits in filter probably maximum chance and could be exchanged with other character key available on keyboard, for example, with English lower case, uppercase letter, and basic numbers characters. After removing special characters if present, by private filter we get the real 32byte Image hash.

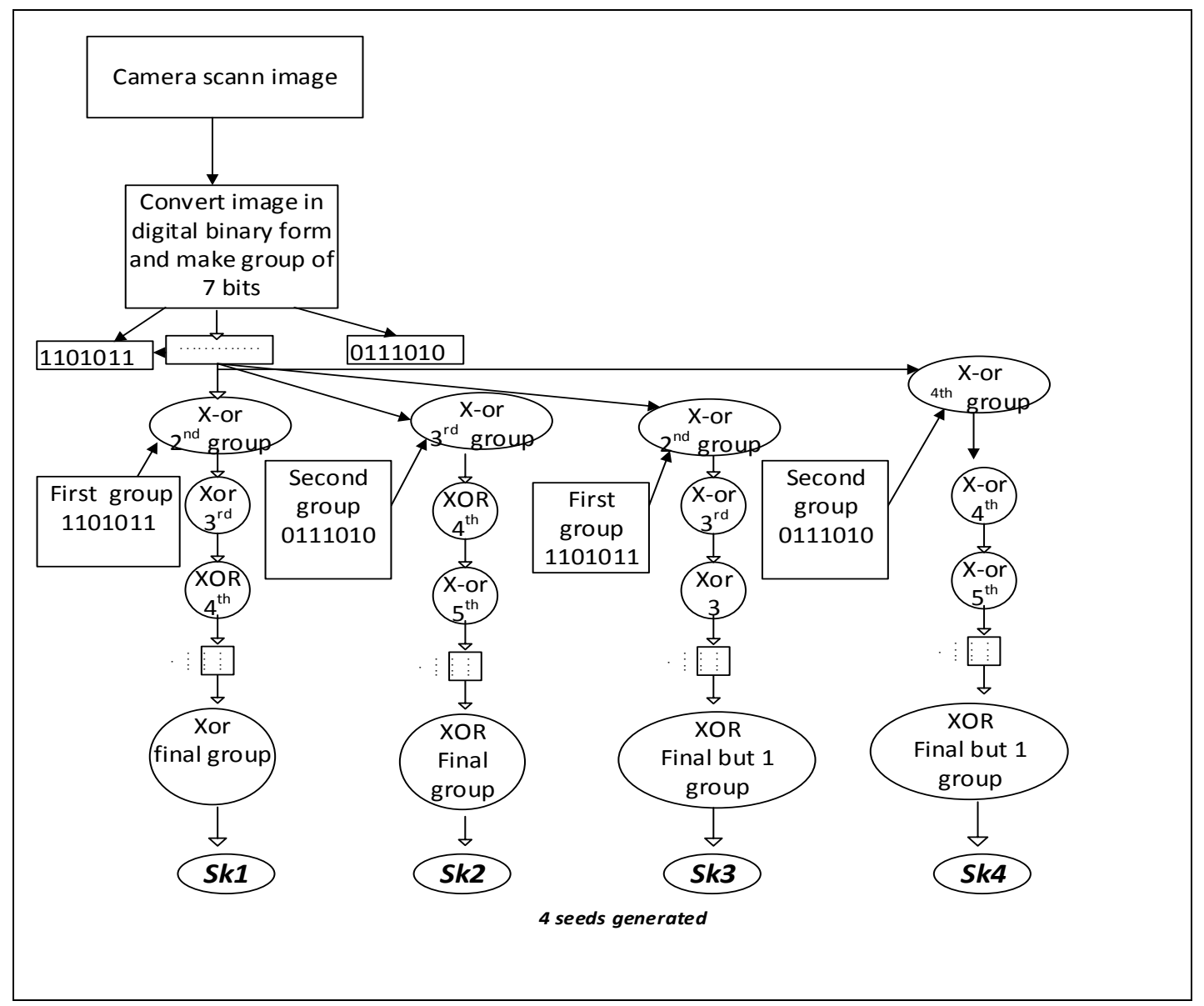

Figure 4: Image Hashing Algorithm Design Flowchart Ph.-I 
International Journal of Engineering Research and Technology. ISSN 0974-3154, Volume 13, Number 9 (2020), pp. 2474-2483

(C) International Research Publication House. https://dx.doi.org/10.37624/IJERT/13.9.2020.2474-2483

Table 2: EfficientImageHashkeyGenerator $(E I H K G)$

Algorithm : EfficientImageHashkeyGenerator $(E I H K G)$

Input: Any image pic

Output:32 Byte Hash key

1. Begin

2. Read or scan an image. (Jpeg or BMP or Tiff or RGB)

3. Convert it into stream of data bits as binary data stream

4. Make a groups of 7 binary bits

5. Pic the groups (it can be sequential or interleaved pickup)

6. Repeat for each group

7. Perform bitwise X-OR operation such that $1^{\text {st }}$ group with $2^{\text {nd }}$ group code and

8. The result with next group till end of groups to get first Seed SK1

9. $\quad$ Repeat for each group

10. perform XOR operation between $1^{\text {st }}$ group and $2^{\text {nd }}$ group and

11. The result with next group till end of groups but 1, to get second Seed SK2

12. Repeat for each character

13. Perform bitwise X-OR operation such that $2^{\text {nd }}$ group with $3^{\text {rd }}$ group

14. The result with next group till end of groups to get first Seed SK3

15. Repeat for each group

16. Perform bitwise X-OR operation such that $2^{\text {nd }}$ group with $3^{\text {rd }}$ group code and

17. The result with next group till end of groups but 1, to get second Seed SK4

18. Repeat $i$ times

19. Generate a $i$ set of 8 random numbers from these seed key Ski where $i=1$ to 4 .

20. Repeat for each set of 8 random numbers

21. Use the Seed key value $S i$ to perform 7 bit XOR operation of $S k i$ with each respective subsequent 8 random number

22. Store the results of each operation in a resultant array of size 32. (Partial Final key).

23. Pass resultant array through secret character filter

24. Filtered key is image hash key value for the given or scanned Image.

25. End 
International Journal of Engineering Research and Technology. ISSN 0974-3154, Volume 13, Number 9 (2020), pp. 2474-2483

(C) International Research Publication House. https://dx.doi.org/10.37624/IJERT/13.9.2020.2474-2483

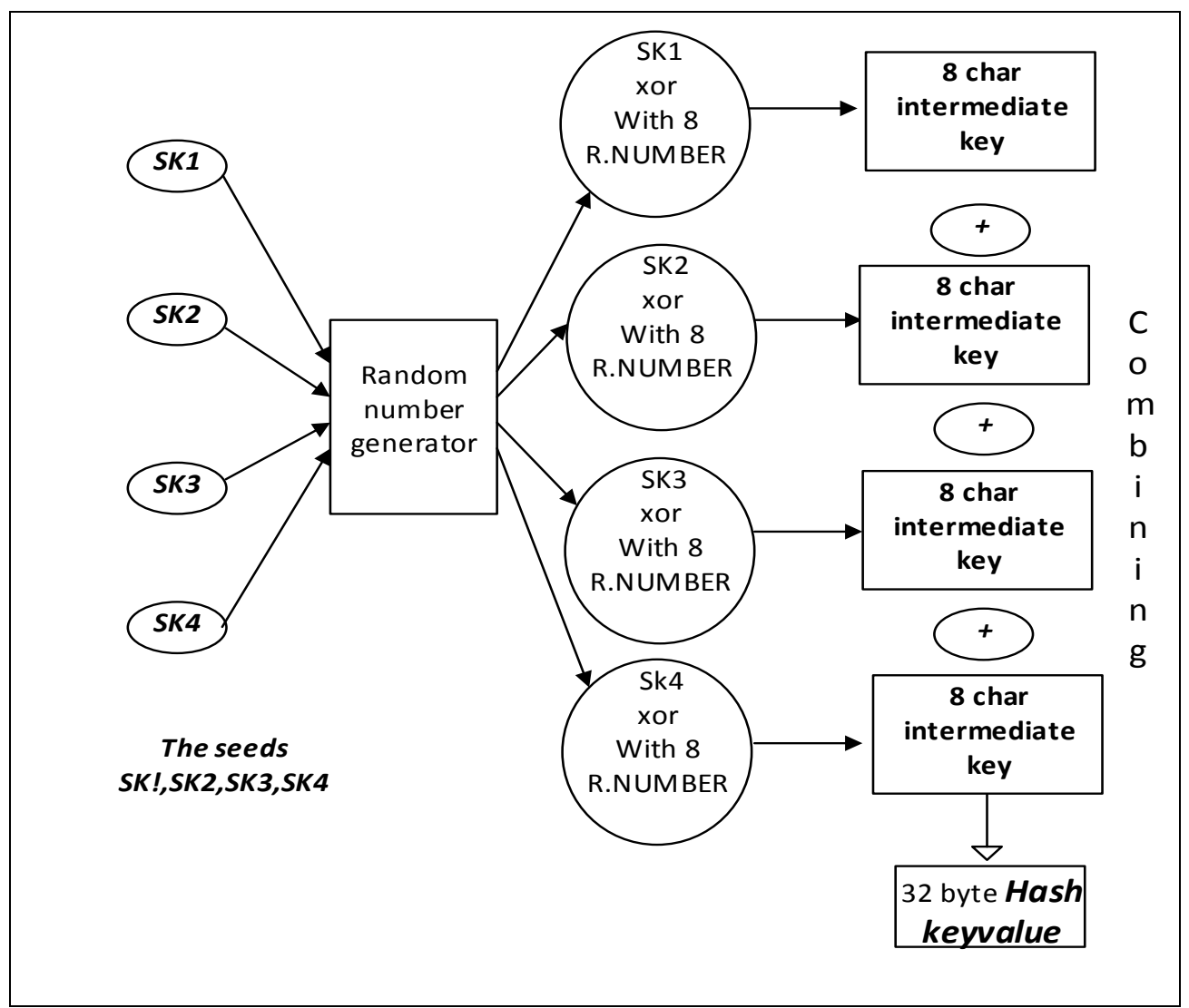

Figure 5: Image Hashing Algorithm Design Flowchart Phase II

\subsection{Proposed Algorithm}

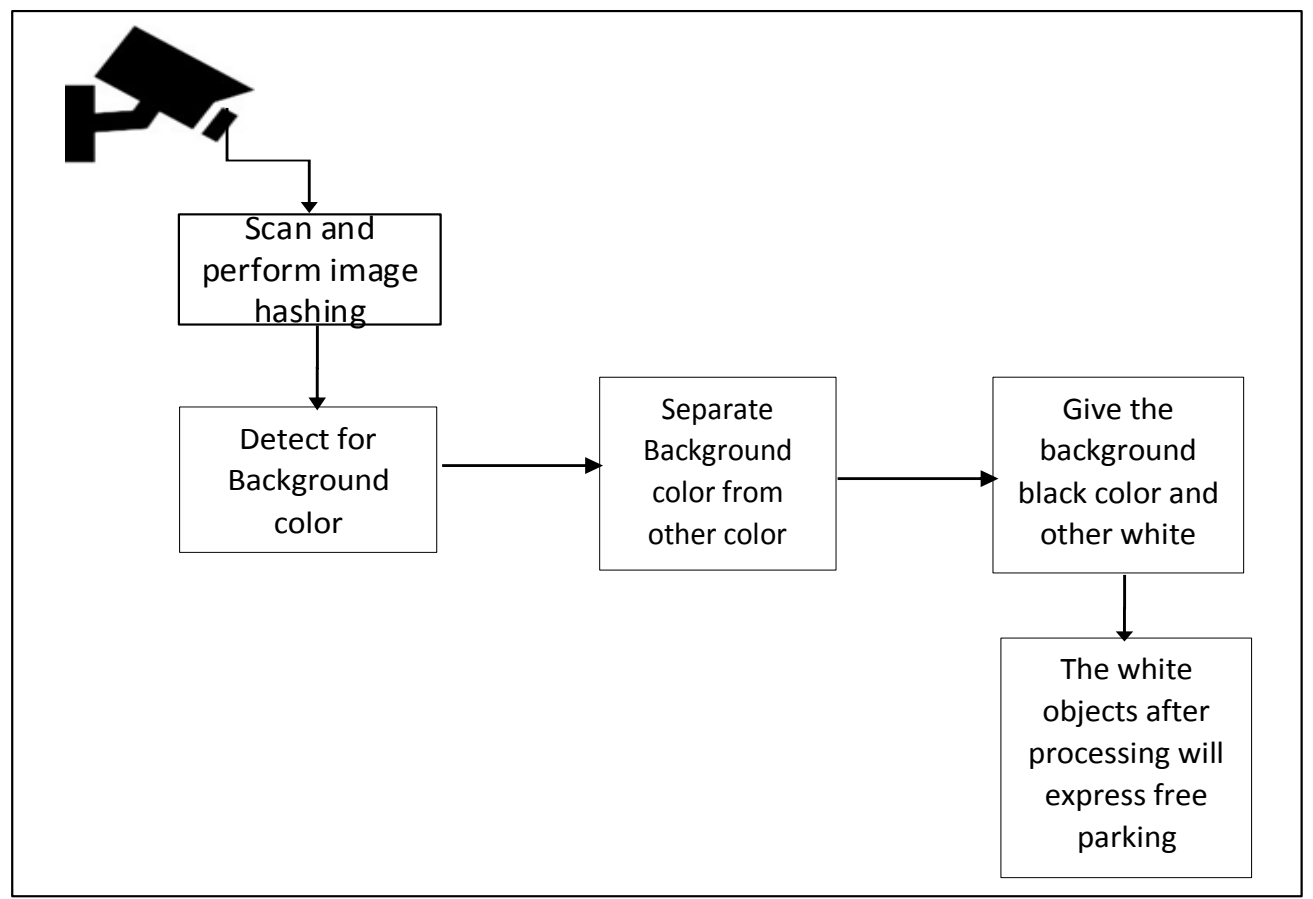

Figure 6: Project proposal model 


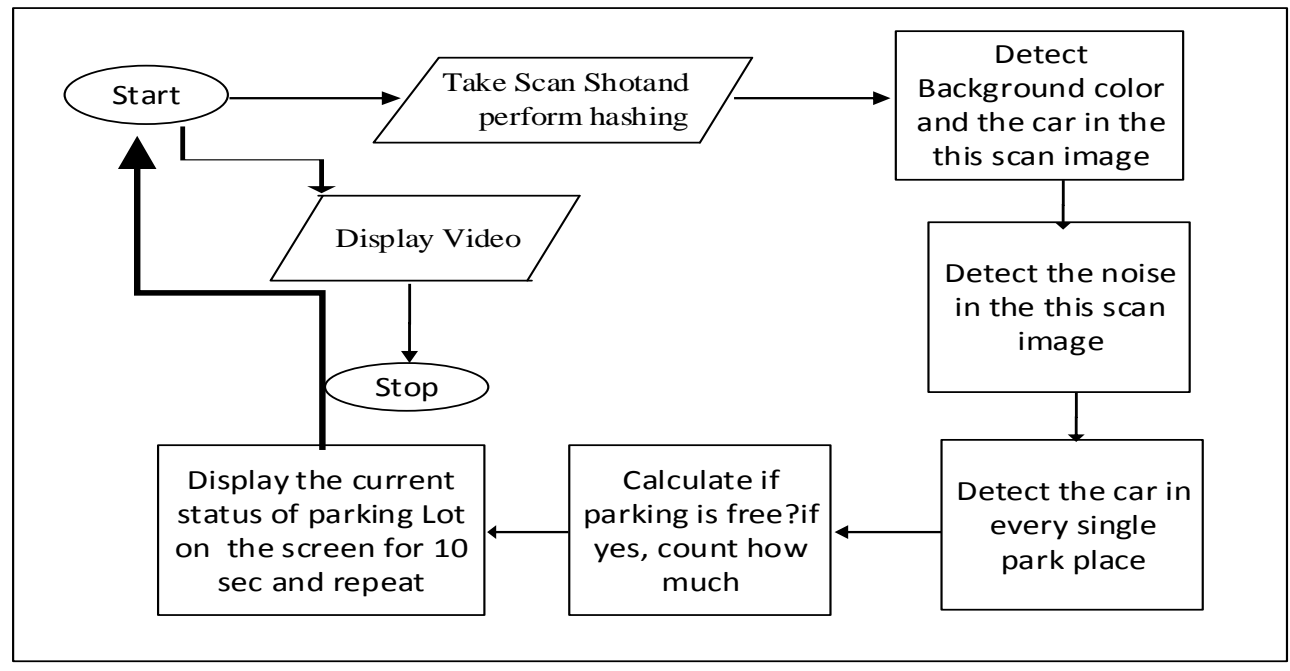

Figure 7: Flowchart

Due to our busy daily life schedule, roaming here and there, we should not waste our time in pretty things like, searching for a parking slot etc. Drivers wasted lot of their time looking for free parking lot. In proposed project we studied that's issue and gave superlative solutions. With consideration, this system must be accessible for all (inexpensive) and can we trust it (reliability) and existing (availability) all time so our system is frugal and use cameras rather than sensors to get high rate of steadfastness. Our proposed solution is to use technique of separate background color for intelligent and well-organized parking. with the help of image hashing technique and MATLAB simulation, the camera will detect and differentiate for background color from foreground colors and texture of image.
The performance of the proposed algorithm is experimented over different range of input parameters, termed as simulation parameters in MATLAB. Our proposed solution is to use technique of placing square on ground for intelligent and wellorganized parking. By the help of MATLAB simulation, the camera will look for the square (parking slot) and this square will be a target or goal for camera to grasp, but the square should having a unique color (for example pink). Then our system will count number of pink squares detected and finally the result will be displayed on the screen. Simulation work of the proposed research work will be carried out in the MATLAB environment.

We present our NovelSecuredEefficientPark Algorithm in Table 3:

Table 3: Proposed pseudo algorithm for efficient parking

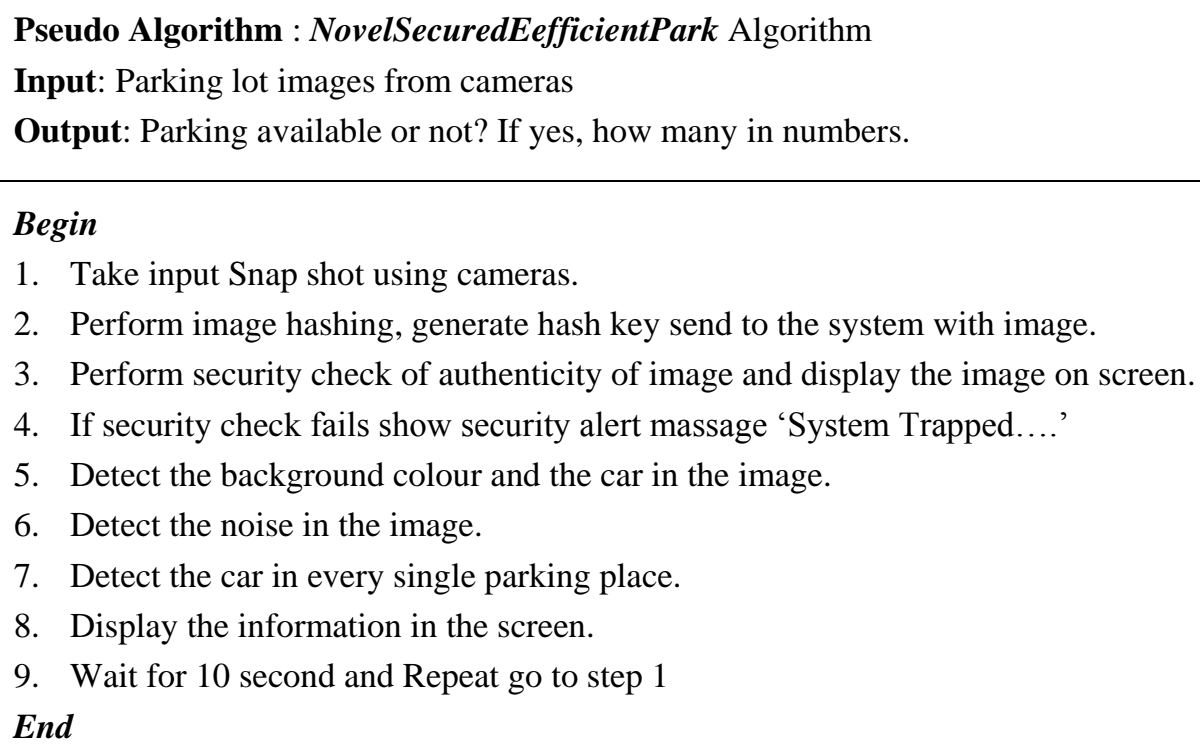

1. Take input Snap shot using cameras.

2. Perform image hashing, generate hash key send to the system with image.

3. Perform security check of authenticity of image and display the image on screen.

4. If security check fails show security alert massage 'System Trapped....'

5. Detect the background colour and the car in the image.

6. Detect the noise in the image.

7. Detect the car in every single parking place.

8. Display the information in the screen.

9. Wait for 10 second and Repeat go to step 1

End 
International Journal of Engineering Research and Technology. ISSN 0974-3154, Volume 13, Number 9 (2020), pp. 2474-2483

(C) International Research Publication House. https://dx.doi.org/10.37624/IJERT/13.9.2020.2474-2483

Our proposed pseudo Algorithm is shown in Table 2. It contains Nine major steps that start by taking Snap shot and performing security check by our newly designed security enhancer image hash generator algorithm of this snap shot. The hashing key is sent along with this snap shot picture to the microcontroller system for further processing and display the video. The next step is detecting the background colour and the car in the image because we mainly interested for separate the background colour from other colours that will be first step in looking to free parking. Then algorithm will detect the noise in the image to clear the car so car look almost clear then we will detect the car in every single parking place to know availability of every parking. Finally, will display the information in the screen that contain number of free parking places. with their positions. The system repeats this cycle in every 10 seconds to keep real time information of parking lot thus to reduce the crowd near the parking entry gate and reduce to form bottleneck on the gate and on the road.

\subsection{SIMULATION SCENARIO AND RESULTS}

The performance of the proposed algorithm has been simulated on MATLAB. To facilitate reader understanding of the two given scenarios in system and how it works, we make graphical representation that shows in two cases.

\subsubsection{Simulation scenarios}

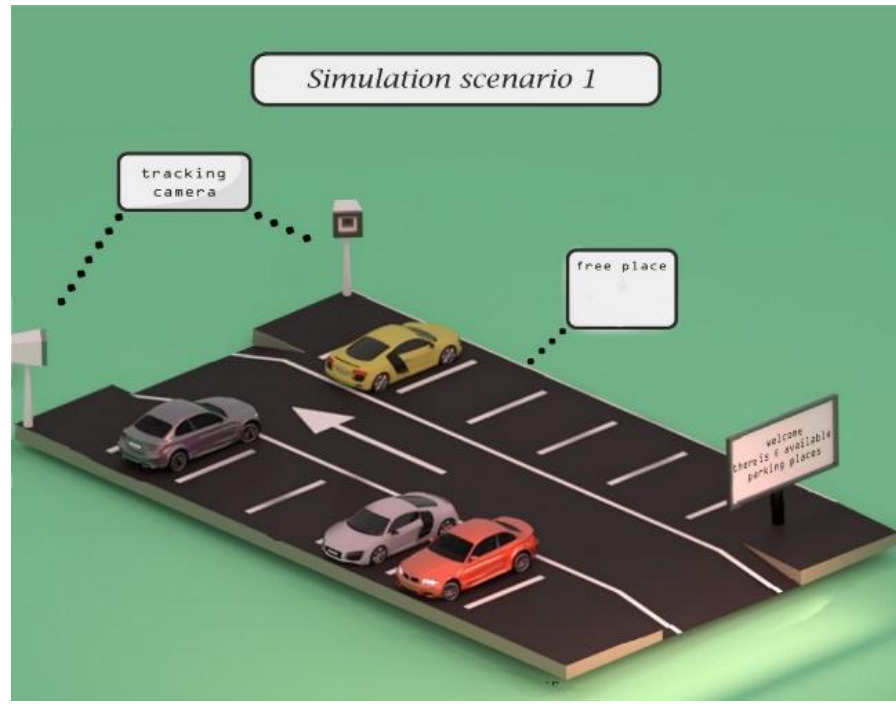

Figure 8: Implementation of novel Parking System Scenario 1

Case-I: First scenarios will show-case us some empty parking places. The screen will show number of empty parking.

As in Figure 8 the cameras are looking for free parking place first, then it finds there is some visible parking places are available so it detects that, so count them through system and shows on the output screen as number of free parking places available with their places.

Case-II: Second scenarios will show if parking is full and there is no place for any car to park, screen will show there is no free parking place available

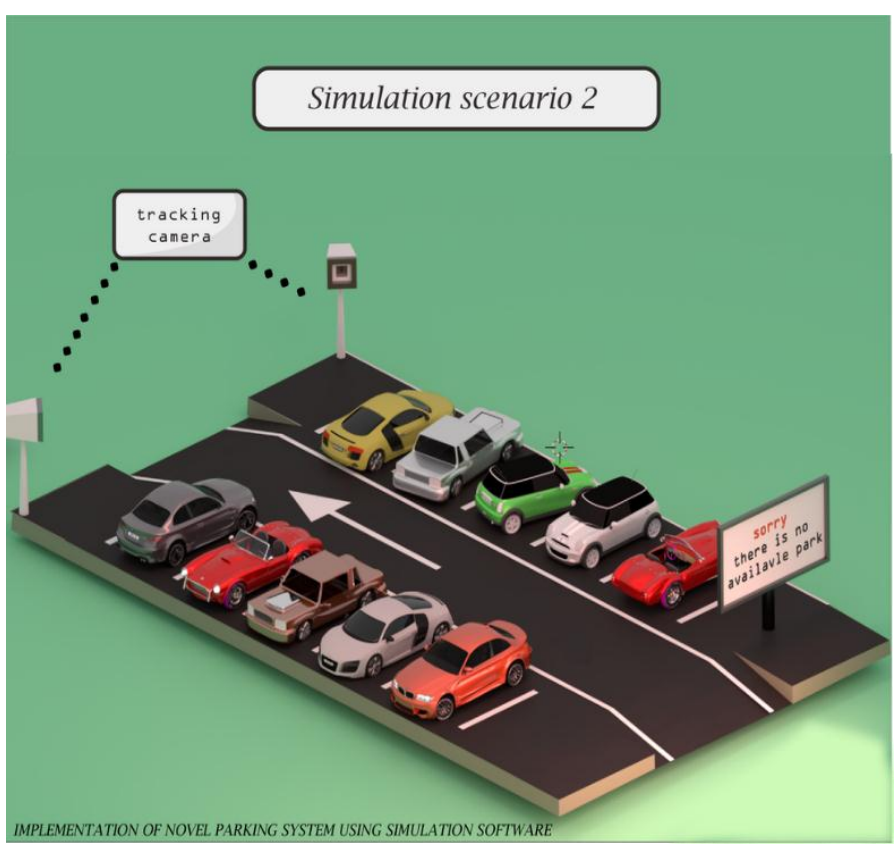

Figure 9: Implementation of novel Parking System Scenario 2

In Figure 9 all the cameras will be looking for any free parking and if it does not found any free parking, system will consider number of free parking place equal Null so the screen will show massage to motorists stating that there is 'Sorry no parking available now...'.

\subsubsection{Verification and validation of system:}

Are we building the system correctly? (need to verification), Are we building the right project? (need to validation). To know the project is right we need to check the specification of system objects. To do validation we cover eight parking places by cars and test our system reaction whether it is giving us correct information about parking lot status or not.

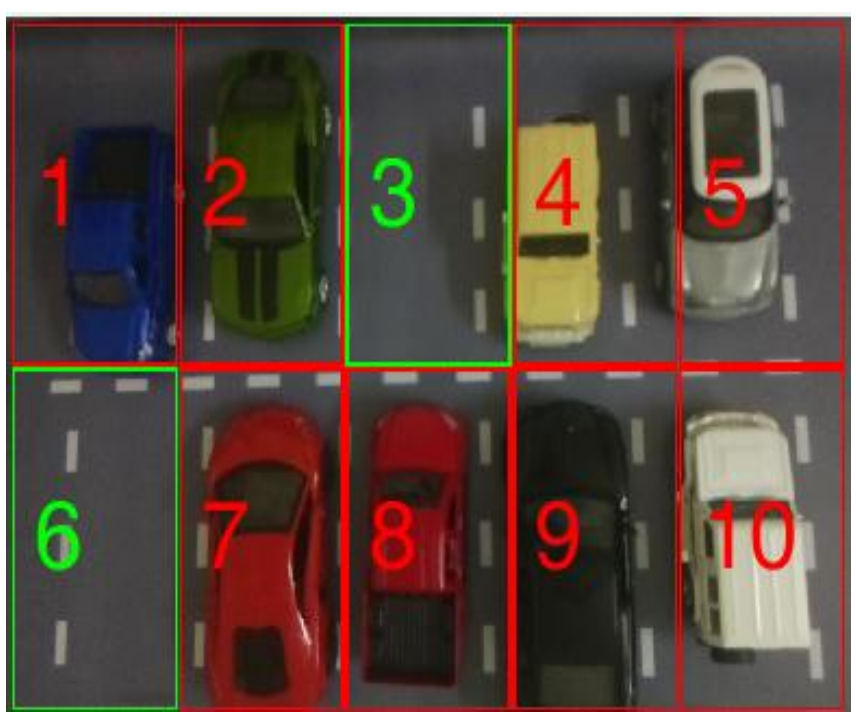

Figure 10: Result from GUI 
As we can see in the figure 10 and figure 11 the system reads data correctly and shows the covered parking boundaries in red color boundaries numbered $1,2,4,5,7$ and 8 , similarly the free available parking boundaries in green color boundaries they are numbered 3 and 6.

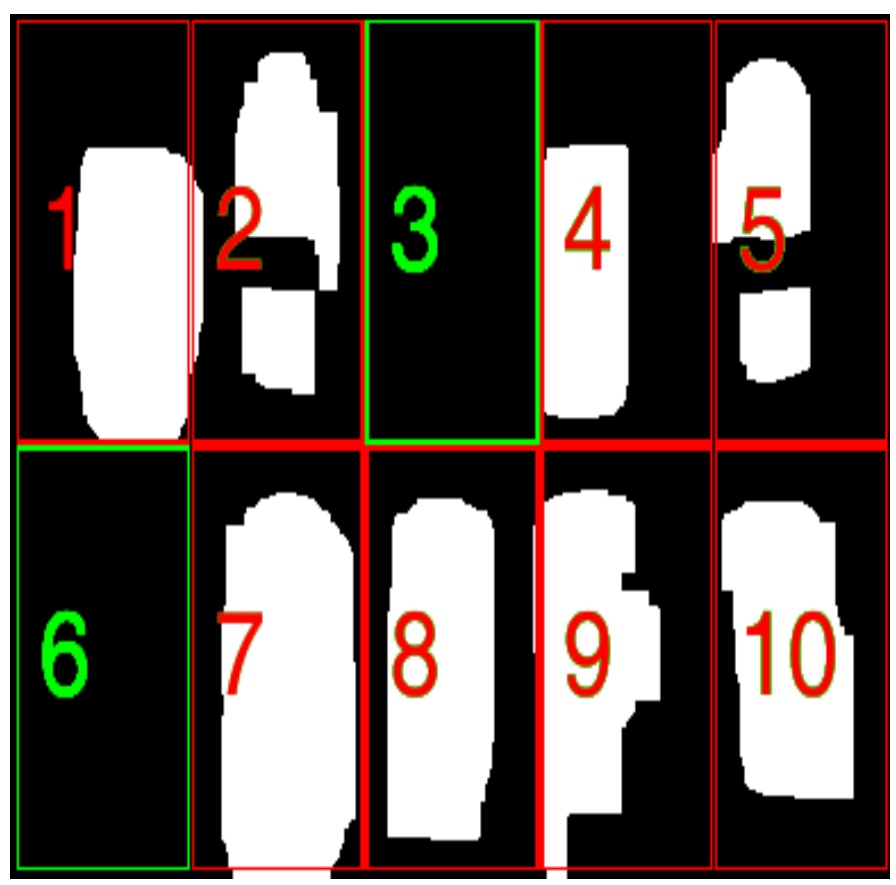

Figure 11: Result black and white

We can see here in figure 11 how system read the data in black and white colors. The parked car takes white color while the back ground takes black color. Sample message on the screen shown in figure 12 shows that how the result will be shown to motorist. This contains information about number of free parking with their position.

\section{There is 2 available free Praking Parking Area A3 B1 Total Cars 8}

Figure 12: Sample message

\section{CONCLUSIONS}

In the proposed work we try to improve the state-of-the-art in security application for automated parking lot application using our newly designed Image hashing algorithm which is capable of generating 32-byte key of almost any digital image. We have developed this proposed work using Matlab Image Processing tools on different simulation scenario of parking lot. We found that the using secured way through cryptographic way of security, results are correct up to the mark and secured for all internal communication in the system. No intruder can distract the system by other image injection in the system. Although there is some limitation and assumption like camera will take bad image quality reading if the light is low or there is reflection of light on ground so it may wrongly count total numbers of cars present in the parking. Also, camera may have wrong reading if the car has exactly same color as of ground, but this scenario chances is very low because difference in image texture material between car and ground will make difference. In future we have plan to improve our work by implementing it using computer vision and machine learning techniques to make our cameras learning behavior of users and make the system behaviors changeable depend on each user.

\section{ACKNOWLEDGMENTS}

This work is supported by Scientific Research Deanship of Qassim University Saudi Arabia under the Project ID: COC2018-1-14-S-3945. The authors are highly thankful of SRD Qassim University for such support. The authors are also thankful to Dr. Arshiya Sajid Ansari for her continuous support throughout this project.

\section{REFERENCES}

[1] "Motorists spend 106 days looking for parking spots", Telegraph.co.uk, [Online]. Available: http://www.telegraph.co.uk/motoring/news/1008246 1/Motorists-spend-106-days-looking-for-parkingspots.html.

[2] Tang, Zhenjun, et al. "Perceptual image hashing with weighted DWT features for reduced-reference image quality assessment." The Computer Journal 61.11 (2018): 1695-1709.

[3] Tang, Zhenjun, et al. "Robust image hashing with multidimensional scaling." Signal processing 137 (2017): 240-250.

[4] Gui, J., Liu, T., Sun, Z., Tao, D., \& Tan, T. (2017). Fast supervised discrete hashing. IEEE transactions on pattern analysis and machine intelligence, 40(2), 490496.

[5] Tang, Z., Li, X., Zhang, X., Zhang, S., \& Dai, Y. (2018). Image hashing with color vector angle. Neurocomputing, 308, 147-158.

[6] Tang, Z., Chen, L., Zhang, X., \& Zhang, S. (2018). Robust image hashing with tensor decomposition. IEEE Transactions on Knowledge and Data Engineering, 31(3), 549-560.

[7] Tang, Z., Huang, Z., Yao, H., Zhang, X., Chen, L., \& $\mathrm{Yu}$, C. (2018). Perceptual image hashing with weighted DWT features for reduced-reference image quality assessment. The Computer Journal, 61(11), 1695-1709.

[8] Kamble, Amit A., and Archana Dehankar. "Review on Automatic Car Parking Indicator System." International Journal on recent and innovation trends in computing and communication 3.4 (2015): 2158-2161. 
International Journal of Engineering Research and Technology. ISSN 0974-3154, Volume 13, Number 9 (2020), pp. 2474-2483

(C) International Research Publication House. https://dx.doi.org/10.37624/IJERT/13.9.2020.2474-2483

[9] Sushma, K., P. Raveendra Babu, and J. Nageshwara Reddy. "Reservation based vehicle parking system using GSM and RFID technology." International Journal of Engineering Research and Applications 3.5 (2013): 495-498.

[10] R. P. FOUNDATION, " www.raspberrypi.org," [Online]. Available: https://www.raspberrypi.org/help/what-is-araspberry-pi/.

[11] R. P. Foundation, "https://www.raspberrypi.org," [Online]. Available: https://www.raspberrypi.org/blog/windows-10-foriot/.

[12] "Cite a Website - Cite This For Me", Seekingalpha.com, [Online]. Available: http://seekingalpha.com/article/3965149-intel-wantsinside-robots-drones.

[13] "Camera Module - Raspberry Pi", Raspberry Pi, [Online]. Available: https://www.raspberrypi.org/products/cameramodule-v2/.

[14] "RPi SD cards - eLinux.org", Elinux.org, [Online]. Available: http://elinux.org/RPi_SD_cards.

[15] "Raspberry Pi 3 Grand Opening | Scargill's Tech Blog", Tech.scargill.net, [Online]. Available: http://tech.scargill.net/raspberry-pi-3-grand-opening/.

[16] "Raspberry Pi FAQs - Frequently Asked Questions", Raspberry $\quad \mathrm{Pi}$ 2017. [Online]. Available:https://www.raspberrypi.org/help/faqs/\#po wer.

[17] Mohammadi, Mohammad Sajid, Syed Sohail Ahmed, and Syed Khizer Khaiser. "Design of Efficient Hashing Algorithm to Enhance Information/Network Security", International Journal of Research in Engineering, IT and Social Sciences, ISSN 22500588, Impact Factor: 6.565, Volume 08 Issue 12, December 2018, Page 131-139.

\section{AUTHORS' PROFILE}

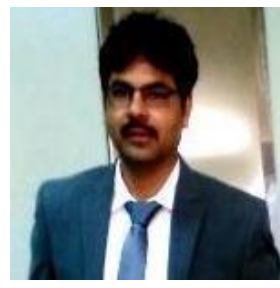

Mr. M. Sajid Mohammadi has completed his B.E degree in Computer Technology from the Yashwantrao Chavan College of Engineering, Nagpur University, India. He did his M. Tech. Computer Engineering from the NMIMS University, Vile Parle Mumbai, India. $\mathrm{He}$ is pursuing his Ph.D. from Noida International University NCR Delhi, India. He has total 16 years of experience including 1.5 years' industrial experience in Reliance Petroleum Mumbai and 14.5 years of teaching experience. He is currently working as Lecturer in Computer Engineering Department, Qassim University Saudi Arabia. His research interest includes Image Processing, Information Hiding, and Information/Network Security. He was a member of Saudi Internet Scientific Society for the year 2017-18.

Mr Sajid: https://orcid.org/0000-0002-3082-5068

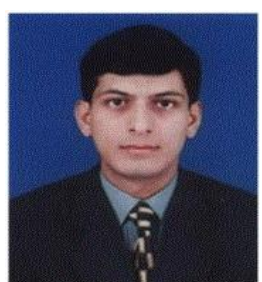

Dr. Syed Sohail Ahmed received the BS and MS degrees in computer engineering from University of Engineering \& Technology (UET) Taxila, in 2005 and 2007, respectively. He did his $\mathrm{PhD}$ degree from University of Engineering \& Technology(UET), Taxila in 2012 with research work from CSIRO, Sydney, Australia. His research interests include wireless networks, digital control, wireless communications and signal processing. $\mathrm{He}$ is a lifetime member of Pakistan Engineering Council (PEC). Currently he is working as an Assistant Professor at Department of Computer Engineering, College of Computer, Qassim University, kingdom of Saudi Arabia.

Dr.Syed Sohail: https://orcid.org/0000-0001-5489-9099

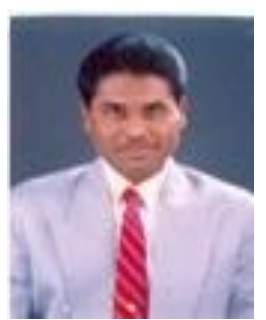

Dr. Syed Khizer is research guide at NIU, India. He has Ph.D. Computer Science (Intelligent Support System), M. sc. (Computer Science) from SRTMU and B.sc (Computer Science) from Dr. BAMU. He is teaching to undergraduate and postgraduate classes for two decades. He has more than decade of strong experience in research. He has publication in international refereed journals. He has successfully completed various research projects funded by different agencies. He is reviewer in international indexed journals. He is actively involved in quality assurance. quality assessment, accreditation and bench marking. His main research interest, expertise and extensive work is in the field of intelligent support system including advisory system and decision support system.

Dr. khizer: https://orcid.org/0000-0002-5984-9637 